\title{
L' écrivain et ses doubles dans le roman contemporain (Christian Mistral et Adriana Lisboa)
}

\author{
O escritor e seu duplo no romance contemporâneo \\ (Christian Mistral e Adriana Lisboa) \\ Eurídice Figueiredo \\ Universidade Federal Fluminense - Niterói - Rio de Janeiro - Brasil
}

$\diamond$

\begin{abstract}
Résumé: Cet article traite de la question de la figuration de l'auteur et de ses doubles dans le roman contemporain au Brésil et au Québec à partir de l'analyse des romans de Adriana Lisboa et de Christian Mistral afin de détecter la présence de l'écrivain lui-même ainsi que de ses prédécesseurs dans des textes qui acquièrent ainsi la forme d'un palympseste. Cette mise-enscène de l'acte de l'écriture brouille les frontières entre fiction et réalité.
\end{abstract}

Mots-clés: Littérature du Brésil; Littérature du Québec; Auteur; Autofiction

Resumo: $\mathrm{O}$ artigo trabalha a questão da figura do autor e seus duplos no romance contemporâneo do Brasil e do Quebec a partir da análise de romances de Adriana Lisboa e Christian Mistral a fim de detectar a presença do próprio escritor e de seus antecessores em textos em forma de palimpsesto. Essa encenação do ato da escrita borra as fronteiras entre ficção e realidade.

Palavras-chave: Literatura do Brasil; Literatura do Quebec; Autor; Autoficção

Parler du roman contemporain est une entreprise périlleuse parce que le critique n'a pas assez de distance de son objet. C'est toujours difficile de discerner quels sont les auteurs qui apportent quelque chose de vraiment nouveau - à savoir ceux qui innovent et contribuent à changer les formes narratives - et ceux qui ne font que répéter les formules créées par d'autres. On ne sait pas non plus si tel auteur qui a publié un premier roman brillant - ou que la critique a encensé - continuera son projet littéraire de manière satisfaisante ou s'il va l'abandonner. Il y a plusieurs tendances dans le roman contemporain et il serait sans doute impossible d'en faire le tour.

Un élément qui me paraît constitutif du récit contemporain est la réflexion sur l'acte même d'écrire, que ce soit sur la figure du créateur et ses doubles, sur les procédés de mise-en-abyme ou encore sur les lectures de l'auteur. Ainsi l'écrivain se situe d'emblée dans une certaine tradition littéraire, se choisit ses prédécesseurs, établissant une intertextualité plus ou moins explicite. Cependant ce niveau méta-littéraire n'a rien de nouveau, il est inhérent à la tradition romanesque depuis $\mathrm{Le}$ Quichotte, si l'on veut bien accepter ce jalon comme étant le premier roman moderne. Dans une esthétique baroque, Cervantes se servait déjà de tous les jeux spéculaires, de tous les dédoublements, y compris la référence au plagiat - au Quichotte apocryphe publié sous le nom d'un certain Avellaneda en 1614.

Si l'objet de la littérature est la littérature elle-même et si l'auteur a toujours joué sur les composantes à la fois autobiographiques et fictives pour écrire des romans, quelle pourrait bien être la nouveauté? Si Cervantes se sert de sa vie pour raconter l'histoire du captif (à partir du chapitre XXXIX Donde el cautivo cuenta su vida y sucesos), si Flaubert affirme que "Madame Bovary c'est moi", si Rimbaud énonçait la présence de l'autre à l'intérieur de soi dans le "Je est un autre", quelles sont les nouvelles formes utilisées par les écrivains contemporains pour traiter la question de l'écrivain et ses doubles? Fredric Jameson considère le pastiche comme l'élément le plus omniprésent dans la production textuelle postmoderne. Le pastiche, différemment de la parodie qui caractérisait le modernisme, est une imitation stylistique sans le rire et la provocation; ce serait comme une parodie blanche. L'imitation des styles morts du passé, l'utilisation de masques qui font partie "du musée imaginaire d'une culture qui est devenue globale" (JAMESON, 2007, p. 44-45), rend la littérature contemporaine plutôt fragmentaire et non-monumentale. Le passé perd son 
rôle d'explication généalogique d'une histoire nationale et devient "une vaste collection d'images" dont se sert l'artiste postmoderne.

L'utilisation de la figure do double dans la contemporanéité me paraît une expression de ce pastiche dont parle Jameson car l'écrivain à la fois cannibalise ses prédécesseurs et se travestit dans un jeu de miroirs qui crée l'illusion d'une série de gémeaux. Selon FernándezBravo,

la littérature a vocation à mettre en scène le double en renversant le principe d'identité: ce qui est un, est aussi multiple, comme l'écrivain en fait l'expérience [...] Par la poétique du double, des écrivains contemporains libèrent leurs héros, qui souvent sont des doubles d'eux-mêmes, de la prison d'un moi particulier, fixé dans le moule de la personnalité (1988, p. 523).

Depuis Les confessions de J. J. Rousseau, la figure de l'ego scriptor, à savoir l'écrivain qui se regarde écrire, est devenue un topos des écritures de soi. Comme dit le narrateur de Christian Mistral: "L'oeuvre est peut-être même [...] tout entière dans le travail qui s'accomplit et s'observe s'accomplir, plutôt que dans la masse morte du produit fini" (MISTRAL, 2004A, p. 329). Or dans la fiction postmoderne - et plus particulièrement dans la série Vortex Violet de Mistral et dans le roman Um beijo de Colombina d'Adriana Lisboa qui seront l'objet de mon analyse dans ce texte - on peut remarquer une prolifération vertigineuse de jeux spéculaires dans lesquels les auteursnarrateurs-personnages se dédoublent.

\section{Christian Mistral}

Le premier roman de Christian Mistral ${ }^{1}$, Vamp (paru en 1988) a été suivi par trois autres: Vautour (en 1990), Valium (en 2000) et Vacuum (en 2003), qui forment la série intitulée Vortex Violet. Dans les quatre romans le personnage-narrateur porte exactement le nom de l'auteur, ce qui peut amener le lecteur à confondre fiction et réalité. Selon Philippe Gasparini, le roman autobiographique se définit par sa "politique ambiguë d'identification du héros avec l'auteur" car le texte suggère de les confondre tout en fournissant tous les indices de fictionnalité. Ainsi l'attribution d'une dimension autobiographique est le

\footnotetext{
Le site officiel de l'auteur fournit des biorepères qui aident à corroborer l'impression qu'il s'agit bien de l'autofiction, c'est-à-dire, que son oeuvre est franchement inspirée par son autobiographie. Né à Montréal en 1964, d'une mère célibataire, il changera de nom lorsque, à la suite du mariage de sa mère, Monique Boucher, avec Réjean Roy, en 1967, il est adopté par son beau-père. Alors qu'il s'appelait Paul-André, il est rebaptisé Christian. Plus tard, lorsqu'il devient écrivain, il adopte le Mistral, un nom de plume, donc. A 16 ans il épouse Natali Tremblay, qui lui donnera un fils - Jean-Christian Hector - l'année suivante. Le mariage ne dure pas. Ces éléments biographiques, mis en récit dans la série Vortex Violet, constituent la charpente de son oeuvre.
}

résultat d'un acte de lecture. "Les éléments dont dispose le lecteur pour avancer cette hypothèse ne se situent pas seulement dans le texte, mais aussi dans le péritexte, qui entoure le texte, et dans l'épitexte, c'est-à-dire les informations glanées par ailleurs" (GASPARINI, 2004, p. 32)

Malgré le décalage temporel entre les quatre romans (15 ans entre le premier et le dernier) et les changements formels dont le plus saillant se trouve dans le dernier, Vacuum, qui est en forme de journal intime, cette série dépeint le style de vie des jeunes gens nés après la Révolution Tranquille, plutôt apolitiques, qui n'avaient plus de compromis avec des idéaux nationaux et se sentaient très proches de l'Amérique, cultivant une culture underground où la figure de Jacques Kerouac se détache parmi d'autres écrivains des Etats-Unis comme Miller, Hemingway et d'autres. La culture de masses américaine, qui va de la musique - notamment le rock - à la télévision et au cinéma, est fortement présente.

Une première caractéristique de la littérature pratiquée par cette génération c'est la présence de la ville de Montréal dans ses romans. La première phrase du chapitre intitulé "Mythomanie verbomotrice" est justement: "J'aimais Montréal, j'en étais fou" (MISTRAL, 2004A, p. 19). La ville occupe une place de véritable personnage de Vamp (et d'autres romans de la dernière décennie du XXème siècle), une sorte de monstre proteïforme qui inspire les jeunes gens vagant dans ses rues.

Montréal riche, blanche, triomphante, de foudre et de soleil. Un coeur qui bat comme une enseigne de néon. Publicité, fast-food, cinémas pornos, voix verte et tonnante de l'argent qui trébuche. S'appartenir, marcher le siècle en rêvassant les poings dans les poches. Montréal amplificatrice des idées et des modes. (MISTRAL, 2004A, p. 69)

Cette ville "riche, blanche et triomphante" qu'il décrit sert de cadre pour cette génération qui boit et se drogue, qui n'a plus de repère ou d'utopie national(iste), qui vit le présent jusqu'à la folie faisant ainsi écho aux poètes visionnaires ou voyants qui l'ont précédé. La flânerie permet au narrateur de décrire la ville presque comme un guide touristique riche de l'histoire et de la poésie qu'elle contient. Lorsqu'il s'arrête au "Château Ramezay, où Nelligan fit son triomphe en 1899 en déclamant sa Romance du vin" (MISTRAL, 2004A, p. 21), le narrateur se met sous l'égide du grand poète québécois et en même temps dans le sillage des poètes français notamment de Musset, Lautréamont, Baudelaire, Rimbaud et de la beat generation américaine. "C'était la génération vamp, née de la Haute Technologie, qui dormait sur un futon, cultivait des bonsaïs, n'allait pas 
à la messe et se torchait une poésie du laid, du bas et du sale parce que sa pauvreté n'entraînait pas qu'elle soit insensible" (Mistral, 2004A, p. 28). En effet, la prose poétique et lyrique de Vamp frappe par son ton rebelle. Selon Mistral il a d'abord écrit de la poésie mais ensuite il a fondu ses "poèmes en une prose lyrique incandescente" (MISTRAL, 2006, p. 164). En un ton fanfarron, lui et son alter ego Blue Jean, affirment une conception de l'art comme performance amoral, dont le premier acte peut/doit être un meurtre (individuel ou collectif) afin de pouvoir apprécier la putréfaction des corps. D'ailleurs le geste surréaliste par excellence n'était-il pas celui de sortir dans la rue un pistolet à la main tirant sur la foule?

Je suggère que l'on pourrait [...] verrouiller les portes coulissantes d'un wagon de métro à demi plein, le détacher du convoi et le transporter tel quel dans le hall d'honneur d'un musée d'art contemporain. Wow! [...] Imagine tous ces gens, tous ces animaux sociaux luttant pour survivre, le vernis de cinq mille ans de civilisation qui se fendille en cinq heures, tous ces rats bien élevés, bien dressés, dans leur cage de verre et d'acier, qui se sautent à la gorge, paniqués, se dépouillent et s'entremangent jusqu'au dernier, et dehors, pressée aux fenêtres, la foule haletante des amateurs d'art en habits de soirée qui contemple l'oeuvre! (MISTRAL, 2004A, p. 106-107).

Pastichant le Rimbaud d'Une saison en enfer, Mistral se dépeint en fin de route, abandonnant la poésie et dénonçant ses sophismes dans Vamp: "Je plantais mes sophismes comme des crocs dans la chair de l'innocence, molosse enragé aux babines écumantes, je voulais me repaître de vieillards faibles et chenus dans mon âpre dégoût de tout ce qui gît immobile" (MISTRAL, 2004a, p. 211).

Etablissant sa généalogie dans "Prémices", le chapitre d'ouverture de Vamp, il pastiche la forme biblique "Et Nanane engendra Christian Mistral. Voilà, enfin devoilée, la trame de mon antique et noble lignage" (MISTRAL, 2004a, p. 15). Son nom évoque le vent de la région de la Méditerranée, froid et violent, appelé Mistral en Provence et sur la Côte-d'Azur et Tramontane dans le sud-ouest de la France. D'ailleurs dans sa généalogie il y plusieurs mots qui désignent les vents: Zéphir, Alizé, Sirocco, Tramontane, Aquilon. Parmi les ancêtres il faut citer Simoun, nom qui est à l'origine du mot simulacre, la marque par excellence du postmoderne. Dans la conception de Platon, simulacre est "la copie identique de quelque chose dont l'original n'a jamais existé" (apud JAMESON, 2007, p. 45).

Outre cette généalogie officieuse, il y en aurait une autre, l'officielle, d'auto-engendrement: "je me suis engendré moi-même [...], [je suis] mon propre père, mon propre fils et mon propre Saint-Esprit. Selon une source généralement bien informée, je serais plutôt le fruit d'une immaculée conception" (MISTRAL, 2004a, p. 11). Christian ainsi est-il assimilé au Christ (MISTRAL, 2004a, p. 192), ce qui confirme le titre du chapitre qui annonce sa "mythomanie verbomotrice". Comme remarque Régine Robin, dans la "recherche d'une identité pluralisée par les fantasmes d'auto-engendrement, il existe une zone limite, une bordure où le passage à l'acte tend à effacer les frontières entre le monde fantasmatique de l'auteur et le réel sociobiographique" (ROBIN, 1997, p. 16). Ces passages entre les frontières menacent l'équilibre instable de l'auteur qui se dédouble infiniment tel un Protée; ainsi Mistral note dans son Journal que "K parle de schizo-fiction et il a bien raison. Annie dit que j'écris plein de menteries et elle n'a pas tort" (MISTRAL, 2003, p. 79). Le dévoilement de cet aspect schizo signale que le personnage-narrateur-auteur fait jouer tous les autres qui sont en lui et en même temps se laisse transformer en autre, devenant ainsi sa propre fiction. Pour écrire une autobiographie, selon Mikhaïl Bakhtine, l'auteur doit devenir autre par rapport à lui-même, se regarder avec les yeux d'un autre (BAKHTINE, 2003, p. 13) car "l'événement esthétique, pour s'accomplir, nécessite deux participants, présuppose deux consciences qui ne coïncident pas" (BAKHTINE, 2003, p. 20).

L'essence de l'oeuvre de Mistral est autoréférentielle: le narrateur-personnage, qui porte le nom de l'auteur, écrit toujours à la première personne. Les autres personnages, ouvertement inspirés par ses amis/amies, sont des espèces de doubles du personnage-narrateur-auteur: ils boivent énormément, ils parlent sur leur travail d'artiste (musique, littérature, travail académique), ils écrivent (ou composent ou peignent), ils font l'amour et ils déambulent dans Montréal, surtout dans les bars, ou restent carrément à la maison (surtout dans les derniers romans de la série). A chaque roman, un ou deux amis intimes, sorte de frère qui partage la maison et la vie: Blue Jean et Fantasio dans Vamp, Vautour dans le roman auquel il a prêté le nom, Léo dans Valium et Kevin (et Mario) dans Vacuum.

Le sujet qui parle de son "exil intérieur" (MISTRAL, 2004A, p. 320) dans les romans de Mistral n'est pas un je plein et rationnel; il se dissout plutôt dans la perte de certitudes. Un je plutôt évanescent s'interroge sur cet autre qui se trouve à son intérieur et qui parle à sa place. Le "je est un autre" de Rimbaud devient l'emblème de l'écrivain contemporain qui dévoile l'éclatement du moi, fragile dans la conscience de son incomplétude. Le double de la "Nuit de décembre" d'Alfred de Musset, ce "jeune homme vêtu de noir,/qui me ressemblait comme un frère", cette image d'une Solitude toute allégorique, se transmue en un double plus inquiétant qui peut le rendre fou. 
Parfois [...] je maugréais contre cet autre en moi qui me suivait depuis toujours, ce petit monstre corrosif qui me jetait à l'eau quand j'avais les deux pieds solidement plantés sur la terre ferme et me repêchait juste quand j'allais me noyer [...], il m'arrivait de croire un instant que j'étais foutu, qu'on finirait par s'apercevoir qu'un fou rôdait dans la ville et qu'on m'enfermerait. (MISTRAL, 2004A, p. 161)

Il a le sentiment que la gémellité qu'il partageait avec Blue Jean se transfert à Fantasio. Ce double, qui renvoie au personnage-bouffon de la pièce Fantasio, de Musset (publiée en 1834), n'hésite pas à citer le poète romantique français. En feuilletant le texte de Musset, on peut prendre une réflexion désabusée et blasphématoire sur l'amour, faite par Fantasio, qui est à la même longueur d'onde du mood de Mistral et ses doubles: "L'amour est une hostie qu'il faut briser en deux au pied d'un autel et avaler ensemble dans un baiser; il n'y a plus d'autel, il n'y a plus d'amour. Vive la nature! Il y a encore du vin" (MUSSET, 1964, p. 260). Les doubles littéraires peuvent se confondre avec les doubles de la vie réelle et le portrait que l'écrivain en fait risque de ne pas plaire aux personnes qui les ont inspirés. Mistral affirme dans Origines que l'amitié avec Jean Borduas, qui lui a inspiré le personnage Blue Jean, s'est refroidie après la publication de Vamp. "Là où j'avais voulu dessiner un héros archétypal et rendre hommage à mon ami, lui ne voyait qu'exploitation et secrets trahis" (MISTRAL, 2003, p. 89). Le brouillage entre vérité et fiction apparaît lorsque son ami, quelques années plus tard, croit se souvenir de quelque chose qui apparaît dans le roman et qui était pourtant fictif. "Je l'avais totalement inventé, mais après tout ce temps, il se souvenait de cette fiction avec une tendresse amusée, allant jusqu'à préciser des détails. Le romanesque avait remplacé sa mémoire défaillante, redessinant l'histoire. J'en fus aussi troublé qu'instruit" (MISTRAL, 2003, p. 90). Le lecteur peut bien se demander qui s'est trompé, qui a fantasmé, si Mistral ou Borduas.

Vamp l'a rendu célèbre ${ }^{2}$ mais, selon lui, ce n'est qu'à partir de Vautour qu'il a su écrire, "peut-être ne ferais-je jamais mieux que ce livre-là" (MISTRAL, 2003, p. 57). Après cette période de relative gloire, Mistral passe par une crise provoquée par un procès, sous l'accusation de violence conjugal, et par un séjour en prison, confirmant sa vocation de suivre les traces des poètes maudits. Dans l'intrigue de Valium le suicide d'une de ses maîtresses, Jo Genêt, suivi de l'abandon de ses amis, métaphorise la crise qui a fait suite à son incarcération. Le titre est justifié au dernier chapitre: "C'est ainsi que j'ai pensé au

\footnotetext{
2 Il commence à écrire Vamp à l'âge de 21 ans; le roman est publié par Québec/Amérique, dont le directeur littéraire, André Vanasse, voit en lui "un auteur dépenaillé capable de manier le verbe avec la dextérité des grands magiciens", "enfin un écrivain qui dessille l'oeil de la littérature québécoise du tranchant vif de son stylo acéré" (site officiel).
}

Valium [...]. A tout le moins, elle avait avalé une tisane de valériane par-dessus quelques cocktails. Le Valium vient de la valériane" (MISTRAL, 2004c, p. 373-374)

Le dernier livre de la série Vortex Violet, Vacuum, est un journal intime; le même nom apparaît dans le site officiel de l'écrivain, sous la forme d'un scrapbook. Dans Valium il affirme qu'il ne veut pas pratiquer le journal intime parce que l'on est censé dire la vérité pour la postérité: "N'étais-je pas celui qui pratiquait tous les genres littéraires à l'exception du journal intime, parce que pour bien le faire, il faut arrêter de vivre et raconter sa vie, mais surtout parce qu'on est tenu d'y consigner la vérité, d'y livrer ses mystères, de s'y anéantir à la grande lumière?" (MISTRAL, 2004c, p. 184). Cependant dans Vacuum, au moment où il fait un journal intime, il change d'avis justement parce qu'il considère que dans l'autofiction il écrit pour ses contemporains et par conséquent il n'a pas besoin de dire la vérité.

Ce journal, c'est autre chose. Longtemps je m'y suis refusé, incapable de substituer l'acte d'écrire à celui de vivre, certain que le processus ne pouvait déboucher que sur l'absurde. De plus, je tenais ce genre pour particulier en ce qu'il s'adressait à des lecteurs futurs; or, cela me créait une obligation de dépeindre la vérité, toute la vérité, toute ma vérité, un exercice aussi périlleux que futile auquel je répugnais à me soumettre. Mais le fait de tenir un journal pour des lecteurs immédiats change la donne, à mon sens: c'est le vrai contre la vérité sélective, ou ce qu'on appelle aujourd'hui avec quelque abus l'autofiction. De plus, la seule perspective d'être lu et d'en jeter plein la vue me donne l'impulsion nécessaire à l'ouvrage quotidien (MISTRAL, 2006, p. 67).

Il s'agit d'un livre fragmentaire, constitué de notations variables, plus ou moins longues, avec les précisions de dates et heures, qui vont du 31/03/2002 au 31/12/2002. Il $\mathrm{y}$ a beaucoup de références aux occupations ménagères ou activités quotidiennes telles que: voir des films à la télé, écouter de la musique, lire le courriel, recevoir les amis et bavarder. Il reste au lit très souvent. Mistral met en scène dans Vacuum l'effacement de frontières entre vérité et fiction lorsque ses amis lisent son propre livre (apparemment mis sur internet avant publication) et commentent la manière dont ils sont dépeints dans le rôle de personnages. Kevin trouve que sa vie plutôt "absurde" fait plus de sens dans le texte que dans la réalité:

Je me disais justement, en le relisant, que mes monotypes et ma peinture industrielle, c'est conséquent, c'est toujours moi, je veux dire que ma vie et mes choix ne sont pas aussi absurdes qu'ils m'apparaissent parfois [...] même quand t'inventes mon dialogue, je rechigne pas, mais enfin, y a littéraire et littéraire... (MISTRAL, 2006, p. 128) 
Vivre et écrire, vivre ou écrire, voilà la question quelque peu paradoxale posée par le narrateur qui semble avoir perdu sa capacité de fabulation. "Plus on écrit [...] moins on vit de quoi écrire qui soit autre chose que l'acte d'écrire". De l'autre côté, écrire peut signifier vivre deux fois (MISTRAL, 2006, p. 273). La crise est visible car, comme affirme Fernández-Bravo, "à la jubilation qui accompagne la proclamation de l'effacement du moi par l'appropriation du double [...], correspond toujours le sentiment tragique de la perte du moi et la hantise de la folie chez ces mêmes auteurs" (FERNANDEZBRAVO, 1988, p. 523). Malgré sa verve, il me semble que Christian Mistral se trouve dans une crise de créativité, vide, comme indique le titre de Vacuum, sans la force vitale démontrée dans Vamp. L'écriture pour lui devient un effort, sa capacité fabulatrice semble tarie. "Je devrais travailler mais le coeur n'y est pas, n'y est plus depuis longtemps, des années, or personne ne me croit quand j'affirme préférer laver la vaisselle à écrire" (MISTRAL, 2006, p. 66).

\section{Adriana Lisboa}

Adriana Lisboa ${ }^{3}$ dans $U m$ beijo de Colombina fait un roman palimpseste, qui s'écrit au-dessus de la poésie de Manuel Bandeira, notamment du livre Estrela da vida inteira, outre quelques écrits en prose. De multiples références et citations retirées du monde poétique de Bandeira parsèment le texte fournissant les personnages - les deux Teresa - et quelques paysages, éléments qui apparaissent dès les premières pages. Ce procédé est explicité dans une Note, à la fin du volume. La première référence se trouve dans l'épigraphe, qui explicite l'emprunt du titre du roman Um beijo de Colombina au poète Manuel Bandeira:

A chama que em suave lampejo

A esquálida tez me ilumina,

Não a ateou febre nem desejo

- Mas um beijo de Colombina.

Dans son roman précédent, Sinfonia em branco, elle emprunte le titre à Whistler, dont le tableau qui porte ce nom est la représentation artistique du personnage central, Maria Inês, la femme aimée par le peintre Tomás, qui la voit toujours comme l'incarnation de ce tableau. On peut remarquer que l'art précède/transforme la réalité car Maria Inês ne devient intéressante aux yeux de Tomás que parce qu'elle "est" le tableau de Whistler un peu comme chez Proust: le regard que Swann porte sur Odette

\footnotetext{
3 Adriana Lisboa (née en 1970 à Rio de Janeiro), João Paulo Cuenca, Santiago Nazarian et Verônica Stigger ont été les jeunes auteurs brésiliens choisis dans un concours réalisé à Bogotá en 2007 "les meilleurs 39 écrivains de moins de 39 ans en Amérique Latine".
}

de Crécy change lorsqu'il s'avise qu'elle ressemble à un Botticelli. Ainsi on peut s'apercevoir que Lisboa crée selon le principe intersémiotique et intertextuel se servant de productions artistiques précédentes sans pour autant se mettre en position de subalternité ou d'angoisse d'influence. Ses romans-palimpsestes se construisent en surimpression sur d'autres oeuvres appartenant au domaine de la peinture, de la musique ou de la littérature.

Tous les chapitres de Um beijo de Colombina reçoivent des titres correspondant à des titres des poèmes de Bandeira. Le premier, Maçã (pomme), commence comme un commentaire du petit poème Maçã, dans lequel il est question des pépins de la pomme "Dentro de ti em pequenas pevides/Palpita a vida prodigiosa/Infinitamente" (BANDEIRA, 2007, p. 181). Le poète emploie un mot rare, à première vue incompréhensible, pevides, motif (au sens de phrase musicale) qui revient à plusieurs reprises dans le roman. Selon le poème c'est dans les "petits pépins que palpite la vie prodigieuse infiniment", c'est-à-dire, ces germes de vie sont la possibilité d'engendrement, de prolifération et de perpétuation de l'espèce. Ainsi l'image des pépins à l'intérieur de la pomme fonctionne comme une métaphore des emboîtements présents dans ce romanpalimpseste, et en dernière instance comme métaphore de l'acte de création et de l'appropriation intertextuelle faite par l'auteure.

En ce qui concerne l'organisation narrative du livre, on vérifie que tous les chapitres sauf le dernier (Unidade) sont écrits par un narrateur masculin, conjoint de Teresa, soi-disant morte noyée à Mangaratiba. Dans les déambulations du narrateur, il suit les traces des parcours quotidiens du poète Manuel Bandeira notamment à Lapa - le quartier bohème de Rio - afin d'apréhender une certaine ambiance de l'écriture du roman que Teresa allait commencer et qui serait inspiré par les poèmes de Bandeira. Ainsi on peut remarquer que, comme chez Mistral, la ville occupe une place fondatrice dans le roman; Adriana Lisboa cherche à retracer une certaine géographie urbaine vécue par ses habitants dans leur quotidien, des itinéraires personnels, subjectifs, sans valeur de monument.

Le narrateur apprend rapidement que Teresa n'est pas morte puisqu'il la voit de sa fenêtre, en compagnie d'une autre Teresa. Ainsi perd-il l'assurance: impossible de représenter le réel puisqu'il se trompe; le motif de sa vacuité et de son chagrin s'avère être un leurre. Le roman se construit donc comme représentation de l'acte même d'écrire; les emboîtements et les doubles se multiplient de manière vertigineuse: comme il se sent vide après la mort de Teresa, il découvre le livre de Bandeira, qui va devenir un viatique pour lui. "[...] o livro, Estrela da vida inteira, tornou-se minha estrela. Meu guia de Teresa" (LISBOA, 
2003, p. 19). Alors il décide de commencer à écrire, lui qui n'avait jamais eu de talent ou l'ambition d'écrire quoi que ce soit. "Queria ver se, contando Teresa no território dela, alguma luz se acendia. E agora prossigo, mesmo sem teclado, sem computador, escrevendo à mão" (LISBOA, 2003 , p. 24). Se sentant inepte et inerte, il doit remplir ses jours et ses nuits produisant un avatar de l'écriture de Teresa, même si ce qu'il écrit ne vaut rien et ne sera jamais publié. Ainsi cet homme est assombrado: il est à la fois hanté par une femme/une ombre de la femme qui l'a doublement trompé: elle a forgé une pseudo-mort et a repris sa liaison homosexuelle avec une autre femme (Teresa aussi).

Cependant au dernier chapitre il y a un rebondissement et c'est un narrateur omniscient extra-diégétique qui parle du livre que Teresa écrit. Le point de vue donné par ce narrateur est celui de Teresa, l'auteure non seulement du livre qui s'écrit mais du livre que le lecteur est en train de lire. Ce narrateur explicite ce renversement affirmant que ce roman court ou peut-être cette nouvelle s'appellera A estrela do beco ou Um beijo de Colombina. João, qui commandait les ficelles du récit jusqu'alors mais qui n'avait pas de nom, alors révélé, perd ses pouvoirs puisqu'il n'a plus de voix, et devient juste le conjoint de l'auteure - un Pierrot amoureux, comme il annonçait à son dernier chapitre (Pierrot branco) - et simple personnagenarrateur du roman de Teresa (ou d'Adriana?), manipulé par elle.

Transformou-o em narrador, ele ainda não sabe, ela não quis contar nada sobre o livro até que tivesse terminado. Ele vai tomar um susto. Engraçado seu nome não ter aparecido, não foi de propósito [...]. João não sabe que todos os doze meses que Teresa levou para escrever o livro, ele teve duas vidas, dupla personalidade. (LISBOA, 2003, p. 133-134)

Les jeux spéculaires se brouillent dans cette duplicité de points de vue révélant que l'auteure se plaît à duper le lecteur, qui se trouve mystifié. Il y a aussi un décalage temporel d'un an entre les deux récits: dans le récit de João, Teresa est en train de terminer un roman et fait le projet d'en écrire un autre, inspiré cette fois-ci par l'oeuvre de Manuel Bandeira tandis qu'au dernier chapitre Teresa dit qu'elle vient de finir justement celuici. Dans les deux récits, ils sont d'accord en ce qui concerne les prix littéraires que Teresa/Adriana Lisboa avait gagnés. L'androgynie, topos récurrent de la duplicité de l'artiste, apparaît ici de manière redoublée car Teresa a un double masculin (João) et un double féminin (Teresa), tous les deux des projections fantasmatiques de l'auteure, qui, elle, serait le double du poète Manuel Bandeira.

\section{Conclusion}

Philippe Lejeune affirme, depuis ses premiers livres, que le roman autobiographique, malgré sa longue tradition, était considéré un déclassé par la critique. Vincent Colonna se demande si l'apparition du terme d'autofiction n'a pas servi à relancer un genre qui était non autorisé. "Interdit d'Histoire et de théorie, sans qualité esthétique, ce 'mauvais genre' est difficile à aborder encore à présent, bien que le public [...] n'ait jamais cessé d'en lire et les écrivains, même d'avant-garde, d'en écrire" (COLONNA, 2004, p. 108).

L'autofiction, utilisant les images de doubles, gémeaux et autres jeux spéculaires, témoigne en effet des mutations du roman contemporain dans la mesure où elle permet une double réception, à la fois autobiographique et fictionnelle. Comme remarque Philippe Gasparini, le degré de véridicité n'importe pas tellement. " $\mathrm{C}$ 'est la richesse rhétorique des procédés de double affichage qui devient, à l'intérieur de cette classe de récits, un critère de classement et d'appréciation" (GASPARINI, 2004, p. 14). L'autofiction serait ainsi une métamorphose postmoderne du roman qui ouvre la voie à la prolifération de selfs de l'artiste, comme a très bien perçu Régine Robin.

Faire jouer tous les autres qui sont en moi, me transformer en autre, laisser libre cours à tout processus de devenir-autre, devenir son propre être fictif ou, plus exactement, s'attacher à expérimenter dans le texte le fictif de l'identité; autant de tentations fortes, presque à notre portée et qui sortent à l'heure actuelle du domaine de la fiction. (ROBIN, 1997, p. 16-17)

La fiction d'Adriana Lisboa et surtout celle de Christian Mistral à l'instar de celle de tant d'autres écrivains d'aujourd'hui jouent sur la possibilité d'être lue comme de l'autofiction exposant ainsi l'image publique des auteurs dans cette sorte d'extimité dont parle Philippe Vilain. En effet, si la littérature s'est toujours utilisé des expériences vécues, les formes de l'exprimer ont changé étant donné que la "subjectivité vécue", selon Pierre Ouellet, "possède son historicité, dans la mesure où l'on ne vit pas son identité et l'altérité à soi de la même manière selon les époques socio-historiques ou les territoires géoculturels: il y a un ethos intersubjectif propre à chaque état de la culture" (OUELLET, 2005, p. 21).

\section{Références}

BAKHTIN, Mikhail. Estética da criação verbal. São Paulo: Martins Fontes, 2003.

BANDEIRA, Manuel. Estrela da vida inteira. Rio de Janeiro: Nova Fronteira, 2007. 
CERVANTES SAAVEDRA, Miguel de. O engenhoso fidalgo D. Quixote de La Mancha. Trad. Sérgio Molina. Ed. Bilingüe. São Paulo: Ed. 34, 2002.

COLONNA, Vincent. Autofiction \& autres mythomanies littéraires. Mayenne: Tristram, 2004.

FERNANDEZ-BRAVO, Nicole. Double. In: BRUNEL, Pierre (dir). Dictionnaire des mythes littéraires. Paris: Editions du Rocher/Jean-Paul Bertrand Editeur, 1988.

GASPARINI, Philippe. Est-il Je? Paris: Seuil, 2004.

JAMESON, Fredric. Pós-modernismo. A lógica cultural do capitalismo tardio. Tradução de Maria Elisa Velasco. São Paulo: Ática, 2007.

LISBOA, Adriana. Um beijo de Colombina. Rio de Janeiro: Rocco, 2003.

LISBOA, Adriana. Sinfonia em branco. Rio de Janeiro: Rocco, 2001.

MISTRAL, Christian. Vamp. Montréal: Boréal, 2004a.
MISTRAL, Christian. Vautour. Montréal: Boréal, 2004b.

MISTRAL, Christian. Valium. Montréal: Boréal, 2004c.

MISTRAL, Christian. Vacuum. Montréal: Boréal, 2006.

MISTRAL, Christian. Origines. Québec: Ed. Trois Pistoles, 2003.

MISTRAL, Christian. <http://christianmistral.com/index. html $>$.

OUELLET, Pierre. L'esprit migrateur. Essai sur le non-sens commun. Montréal: VLB éditeur, 2005.

ROBIN, Régine. Le Golem de l'écriture. De l'autofiction au Cybersoi. Montréal: XYZ, 1997.

VILAIN, Philippe. Défense de Narcisse. Paris: Grasset, 2005.

Recebido: 04 de setembro de 2014

Aprovado: 17 de dezembro de 2014

Contato: euridicefig@gmail.com 